\title{
Un projet de microélectronique numérique original : \\ Contrôle autonome d'un micro-drone par caméras externes
}

\author{
Laurent Fesquet ${ }^{1,2} \quad$ Katell Morin-Allory ${ }^{1}$ \\ Robin Rolland-Girod ${ }^{2}$ \\ ${ }^{1}$ Laboratoire TIMA, Grenoble Alpes Université \& CNRS \\ 46 avenue Félix Viallet 38031 Grenoble Cedex \\ 2 CIME Nanotech, Grenoble Alpes université \\ 3 parvis Louis Néel 38016 Grenoble Cedex
}

27 avril 2015

\begin{abstract}
Résumé
Ce papier présente un travail réalisé par des étudiants en deuxième année d'école d'ingénieurs dans le cadre d'un projet de $56 \mathrm{~h}$ sur la conception numérique. L'objectif de ce travail est de programmer une séquence de vol d'un drone en utilisant une carte FPGA, des caméras et un module de communication radio.
\end{abstract}

\section{Introduction}

Dans le cadre de la deuxième année de l'école d'ingénieur Phelma (Physique, Electronique, Matériaux), les étudiants de la filière "Systèmes Électroniques Intégrés" (SEI) doivent réaliser un projet de conception numérique de 56 heures. Le choix du sujet est relativement libre ( algorithme de cryptographie, microprocesseur, filtrage video, synthétiseur midi, ...). Ces projets consistent à mettre en œuvre une architecture de circuit, en considérant la partie matérielle de l'architecture, FPGA ou ASIC. Les aspects logiciels ne sont pas abordés. Dans ce contexte, des étudiants ont souhaité effectuer un projet mettant en œuvre un drone. 


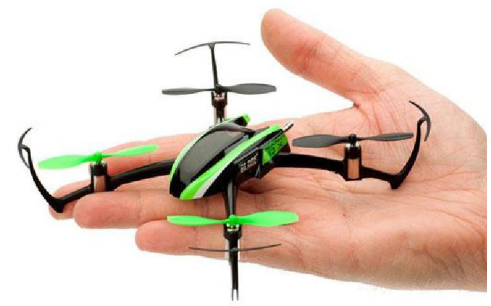

FiguRE 1 - Le drone utilisé

La réalisation d'un drone présente des difficultés techniques qui sont hors du spectre de la microélectronique mais son contrôle peut être envisageable par des moyens matériels spécifiques. Partant de l'idée qu'un drone pourrait être contrôlé par un circuit spécifique, nous nous sommes procuré un drone de taille réduite (NanoBlade QX [2], voir figure 1) afin de limiter tout risque de blessures. Le contrôle du drone est réalisé par une caméra dont l'image est analysée par un circuit implanté sur FPGA [3] qui réalise le repérage du drone dans l'espace et génère des ordres de commandes qui sont transmis par un système radio [1] au drone. Les ordres envoyés permettent de réaliser une séquence de vol prédéfinie. La figure 2 illustre le matériel utilisé.
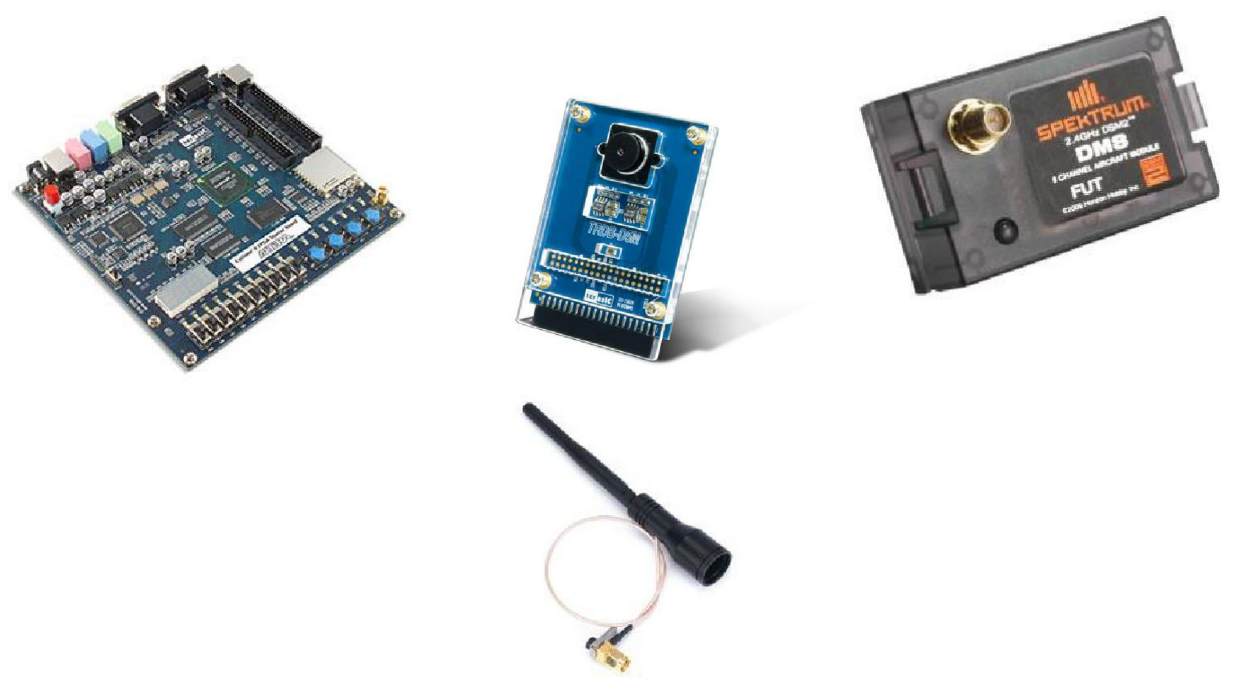

FiguRE 2 - Le matériel nécessaire au contrôle du drone : une carte DE1, une caméra 5 Mpixels, un module radio $2.4 \mathrm{GHz}$ et son antenne. 


\section{Protocole de codage du drone}

Les ordres sont envoyés à un module radio DMSS via une modulation PPM [4] (Pulse Position Modulation). La trame se décompose en 6 canaux de 1 à 2 ms séparés par une impulsion de $0,3 \mathrm{~ms}: 5$ canaux sont utilisés pour le contrôle, le sixième sert à la synchronisation et permet d'avoir une trame de durée constante (22,5 ms).

Les canaux de contrôle Les canaux de contrôle permettent de contrôler les 4 mouvements distincts du drone. Le canal 1 contrôle les gaz, le canal 2 les ailerons, le canal 3 la gouverne de profondeur, et le canal 4 la gouverne de direction (lacet). Un cinquième canal existe pour contrôler le type de vol (stable ou voltige). La figure 3 illustre une trame de commande. Les ordres sont envoyés selon la séquence suivante : contrôle moteur (T1), commande du roulis (T2), commande de la profondeur (T3) et commande de lacet (T4). La durée de chaque canal est proportionelle à l'ordre de contrôle de chaque mouvement.

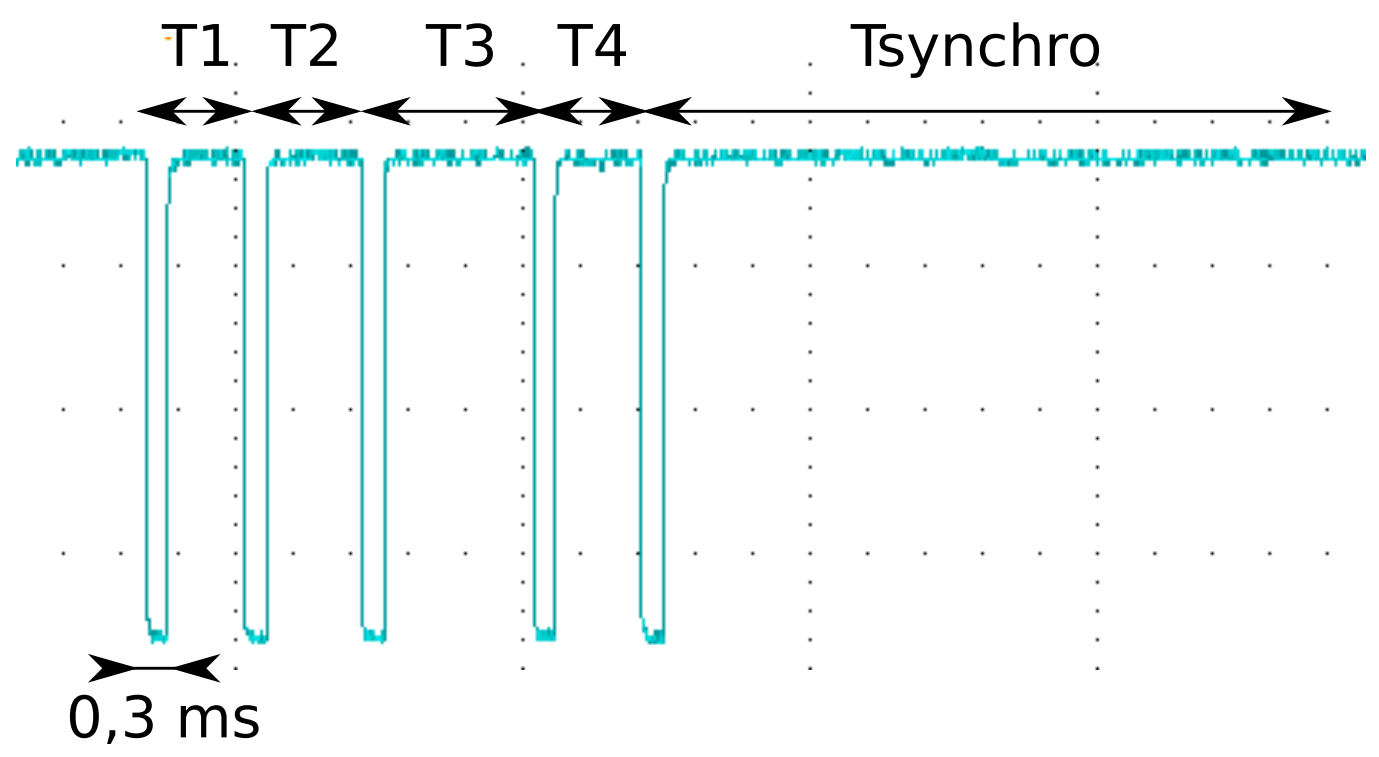

\section{$22,5 \mathrm{~ms}$}

Figure 3 - Protocole de modulation PPM pour le codage des commandes 


\section{Description de l'architecture du circuit}

L'analyse spatiale de la position du drone est obtenue grâce un petit corrélateur réalisant en ligne l'opération sur le flux vidéo. L'information recueillie permet de déterminer l'altitude, la position latérale du drone.

L'architecture du circuit peut se décomposer en 3 blocs principaux :

- un bloc d'acquisition de l'image : "Cam2DPRAM"

- un bloc de traitement de l'image : "Corrélateur"

- un bloc de pilotage : "Processeur de pilotage"

Pour faciliter le debogage, nous rajoutons un quatrième bloc permettant de transferer l'image acquise vers un ecran LCD ( "DPRAM2LCD").

La figure 4 illustre cette architecture.

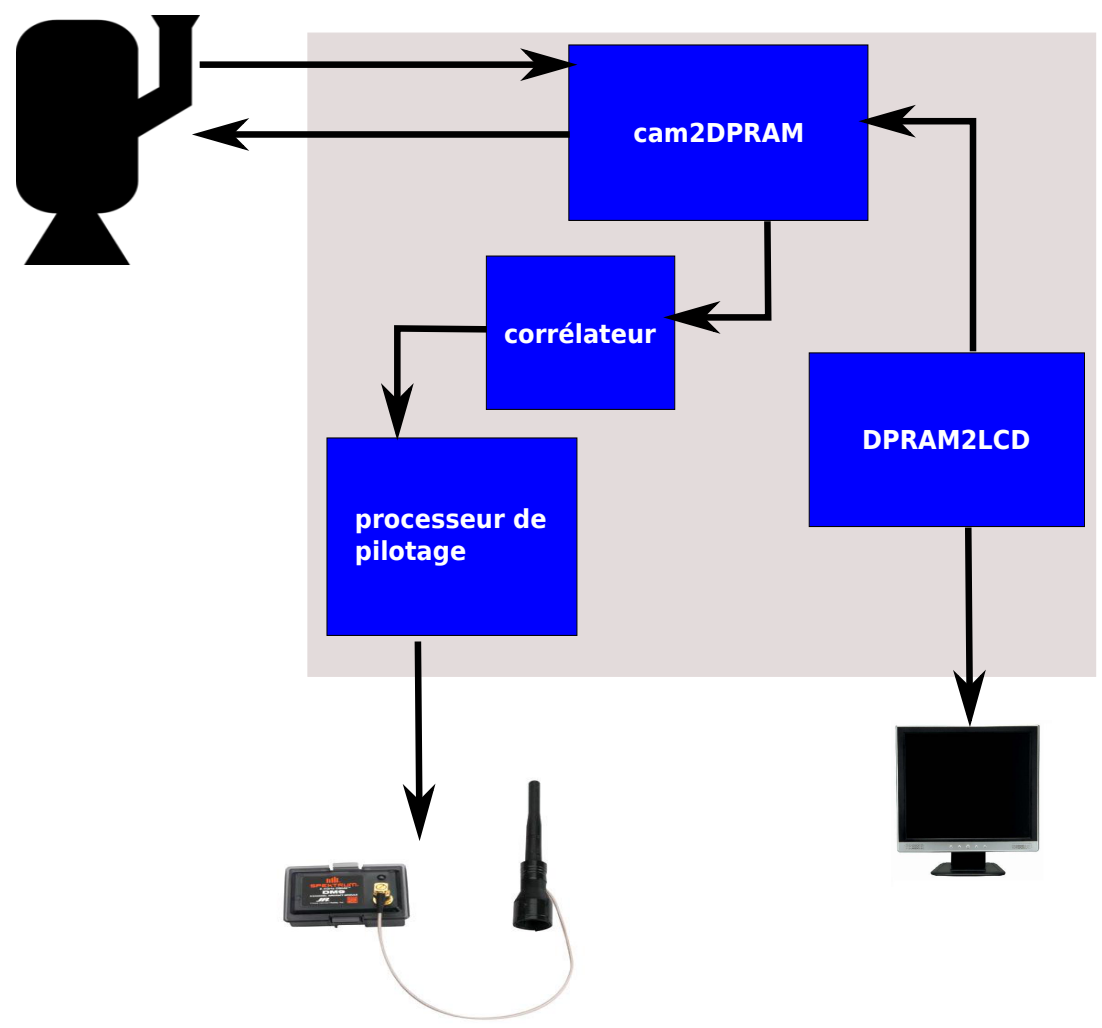

FIgURE 4 - Architecture du circuit

Selon le type de projet souhaité et le niveau des élèves, les blocs de calcul (corrélateur, pilotage) peuvent être implémentés soit de manière logicielle, soit de manière matérielle. Ce travail s'est déroulé dans le cadre d'un projet dédié à la conception d'un circuit intégré ou d'un bloc matériel (IP). À ce titre, les étudiants n'ont pas eu l'occasion de mettre en œuvre une architec- 
ture logicielle. Les projets, à base d'architecture matérielle et logicielle, font l'objet d'un enseignement ultérieur en 3ème année et seront exploités dans les années à venir.

\section{Le bloc d'acquisition de l'image et le bloc de transfert de l'image}

Si les blocs d'acquisition et de transfert de l'image peuvent techniquement être gérés de manière logicielle, cela demande pratiquement une très grande capacité de calcul qui n'est pas compatible avec le projet. Ces deux blocs sont donc implémentés en matériel.

Le traitement de l'image peut être géré en 2D ou en 3D.

Vision 2D Dans un premier temps, nous limitons la profondeur mécaniquement par un mur (en fait on maintient le drone contre le mur par une commande très légère à la profondeur). Cette approche permet de simplifier l'analyse de la scène car elle ne requiert pas une commande en stéréo vision. La position du drone peut ainsi être asservie sur 2 axes (3 si on tient compte du mur!) et une séquence de vol peut être programmée et exécutée avec le drone.

La cadence du flux vidéo caméra dépend du temps d'acquisition d'une image. Ce temps est ajusté via une commande manuelle, l'opérateur ajuste cette valeur selon la luminosité de la scène, et vérifie également que le corrélateur vidéo fournit des coordonnées fiables relatives à la position du drone. Cette cadence caméra varie selon la taille de l'image, ce qui n'est pas le cas de la cadence d'affichage des images sur le moniteur LCD au format VGA $(60 \mathrm{~Hz})$. Un moyen très simple de gérer cette différence de flux est d'intercaler une RAM double port au sein du FPGA. Si la description VHDL est reconnue comme une RAM double port l'outil de synthèse de Altera, Quartus, implémente cette DPRAM, sous réserve que sa taille ne dépasse pas 240 kbit, dans le cas du Cyclone 2 EP2C20 présent sur la carte DE1. La résolution des images traitées est de $128 \times 128$ pixels sur 4 bits par pixel, soit 512 kbit de DPRAM au total.

Le format des images fournies par la caméra est le format "RAW", celui-ci est converti en RGB par le composant HDL "Raw2RGB".

Le composant "Cam2DPRAM" (voir figure 5) orchestre le pilotage de la caméra et l'écriture des images converties en RGB dans la DPRAM. Un composant "DPRAM2LCD" pilote la lecture de la DPRAM et le transfert des pixels vers le moniteur LCD au format VGA. 


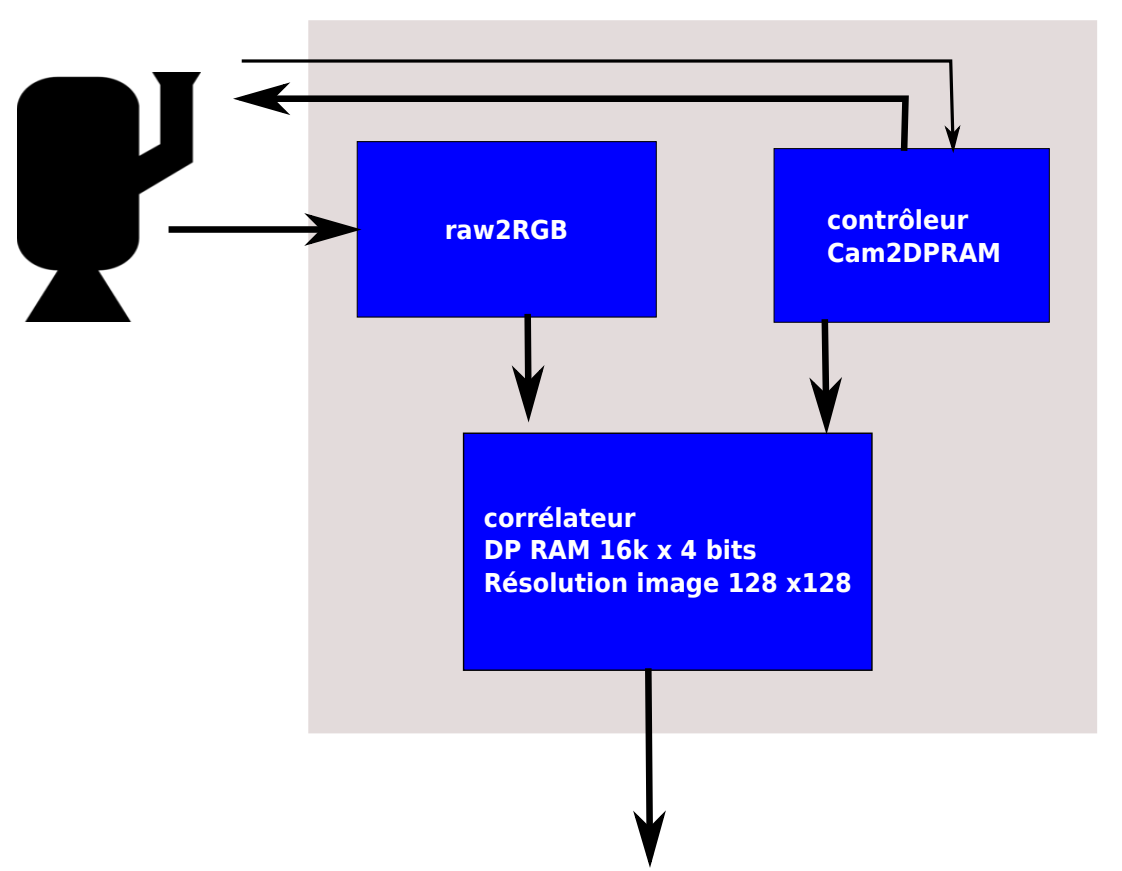

FiguRE 5 - Architecture du composant cam2DPRAM

Vision 3D L'étape suivante, plus séduisante, consiste à contrôler la profondeur à l'aide d'une deuxième caméra latérale qui fournira au sytème de contrôle la position en "Z". Ainsi le drone sera contrôlé dans les trois dimensions de l'espace.

La Caméra 2 est gérée par une carte FPGA qui reprend le bloc de contrôle caméra et le corrélateur, permettant d'extraire la position du drone dans la scène 2D (plan de la profondeur). Ces images binaires sont transmises en temps réel via un bus série $(128 \times 128 \times 1 \times 10=160 \mathrm{kbit} / \mathrm{s})$ si l'on se contente d'une résolution modeste, avec une mise à jour de la position du drone toute les $100 \mathrm{~ms}$ (10 images par seconde, ce qui est suffisant au regard des constantes de temps de la mécanique du vol).

A noter que la caméra 2 est positionnée dans un plan strictement perpendiculaire au capteur de la caméra 1, ce qui nous procure directement une localisation selon un repère orthonormé xyz (voir figure 6). Une vrai stéréovision, utilisant 2 caméras positionnées dans le même plan, conduirait à des calculs beaucoup plus conséquents, pour retrouver la position en 3D.

Le calcul de la position en 3D est réalisé par un bloc matériel dédié, qui procure un affichage vidéo en temps réel (3D ou non, suivant le temps disponible pour le développement du projet). 


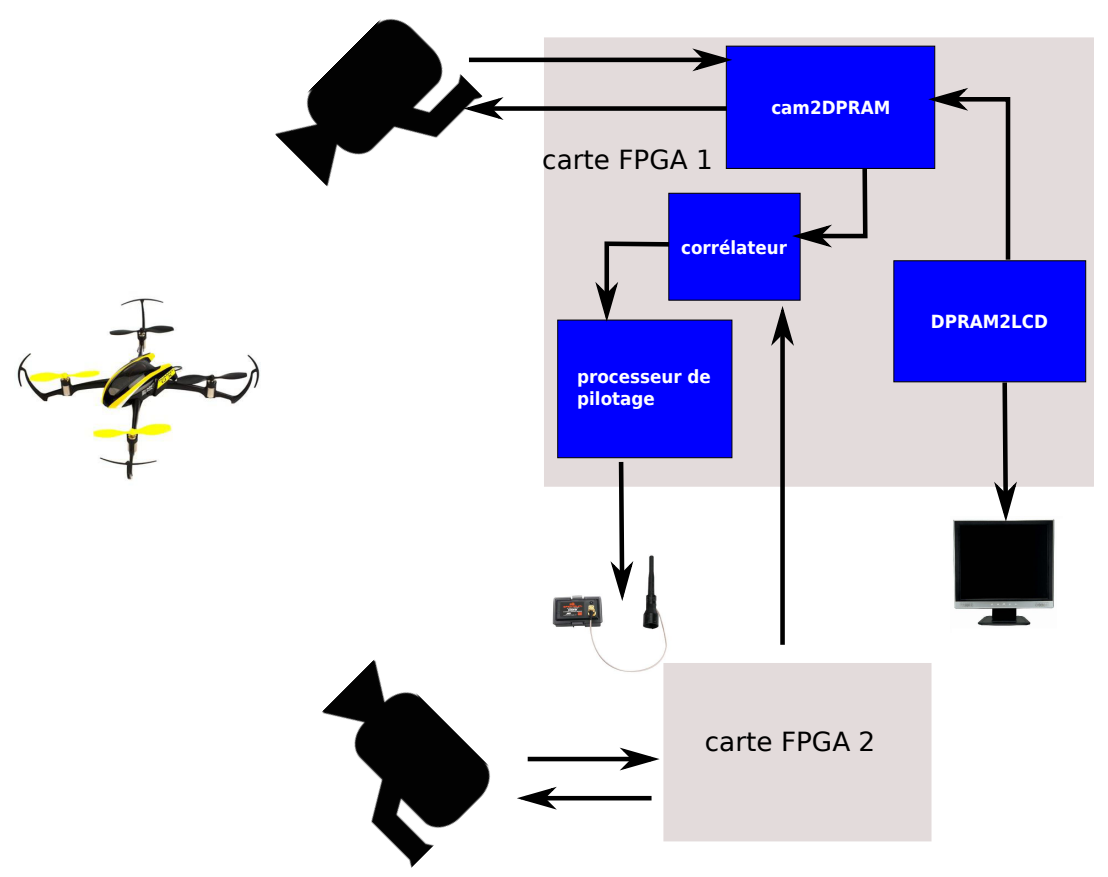

Figure 6 - Architecture du circuit pour la vision 3D

Le bloc corrélateur vidéo La position du drone est analysée en ligne par le composant "Corrélateur".

\section{Processeur de pilotage}

Le composant "Processeur" (7) est l'organe décisionnel qui pilote le drone en fonction de la consigne exprimée au clavier et de la position (telle que visionnée par la caméra) de l'objet volant fournie en format X,Y par le corrélateur. Les informations de pilotage du drone sont encodées en ppm par le composant "Encodeur ppm".

Tout d'abord le processeur va procurer une plus grande souplesse au niveau de la mise au point de la maquette, en substituant une opération de compilation logicielle à des opérations de synthèse et placement routage, nécessaires lors de chaque modification. La simulation fait également partie des opérations incontournables pour valider les blocs les plus critiques de l'architecture. De manière générale, dans un projet de circuit, la logique cablée n'est mise en œuvre que si la logique programmée ne peut répondre aux contraintes, essentiellement temporelles, demandées par le cahier des charges, et ceci en raison de son coût de revient. L'introduction d'une composante logicielle peut par exemple se décliner ainsi. Le partitionnement entre 


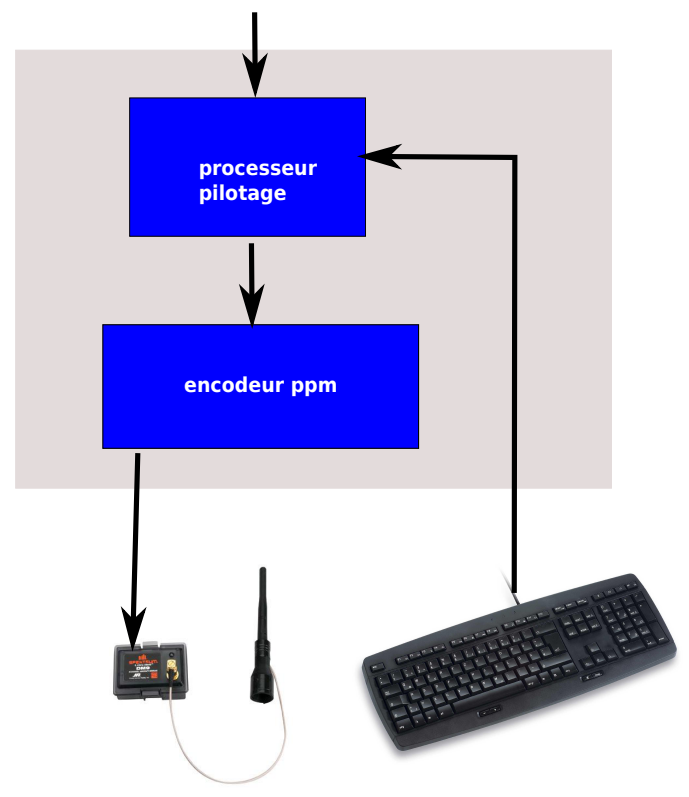

FiguRE 7 - Architecture du processeur de pilotage

matériel et logiciel comprend : le contrôle de la caméra et de la RAM double port, le corrélateur vidéo, la machine à états en charge de l'asservissement de position du drone, l'encodeur ppm ...

$\mathrm{Au}$ niveau du choix du processeur le projet est mis en œuvre sur une carte DE1 équipée d'un FPGA fabriqué par la société ALTERA. La solution la plus simple consiste à utiliser le processeur NIOS2 fourni par ALTERA.

Ce processeur RISC 32 bits, programmable en C, est proposé avec une grande panoplie d'interfaces matérielles (bus SPI, RS232, interface RAM, ...), qui faciliteront la mise en œuvre de l'architecture, sous la forme d'une IP décrite en langage VHDL ou Verilog.

\section{Bilan et Discussions}

L'expérience s'est avérée concluante car elle motive les étudiants par une approche ludique du projet. Elle permet aussi de comprendre les spécificités de l'environnement micro-aéronautique, ce qui permet aux étudiants de partir d'une spécification qui n'est pas complètement fixée. Cela accroit leur travail d'analyse et les obligent à proposer un circuit numérique de traitement du signal vidéo et de génération de la commande PPM suffisamment bien pensé pour être modulaire et flexible afin d'évoluer en fonction des tests de comportement (effectués en parallèle de la conception) sur le micro-drone 
qui est plutôt très vif.

\section{Remerciements}

Ce travail a bénéficié des aides de l'Etat Français au travers des programmes IRT Nanoelectronique portant la référence ANR-10-AIRT-05 et IDEFI FINMINA portant la référence ANR-11-IDFI-0017.

\section{Références}

[1] Air Module System. Spektrum. avril 2015. URL : http://www . spektrumrc . com/ProdInfo/Files/17016-1_DSM2_Air_Module_Manual.pdf.

[2] Blade Nano QX. Fliterc. avril 2015. URL : http://www.e-fliterc. com/ProdInfo/Files/BLH7600-Manual_FR.pdf.

[3] DE1-SoC, User Manual. Altera. avril 2015. URL : ftp://ftp.altera. com/up/pub/Altera_Material/13 .1/Boards /DE1-SoC/DE1_SoC_ User_Manual.pdf.

[4] Y. FujIwara. "Self-Synchronizing Pulse Position Modulation With Error Tolerance". In : Information Theory, IEEE Transactions on 59.9 (sept. 2013), p. 5352-5362. ISSN : 0018-9448. DOI : 10.1109/TIT. 2013. 2262094. 